\title{
Capacidades de innovación en las organizaciones de salud. Propuesta de un modelo mediante ecuaciones estructurales
}

\author{
HERNÁN LÓPEZ, ÓSCAR \\ Facultad de Ciencias Económicas y Administrativas \\ Universidad del Tolima (Colombia) \\ Correo electrónico: ohlopezm@gmail.com \\ VILLEGAS A., GLADIS CECILIA \\ Facultad de Ciencias Económicas y Administrativas \\ Universidad de Medellín (Colombia) \\ Correo electrónico: gcvillegas@udem.edu.co
}

\begin{abstract}
RESUMEN
El presente trabajo pretende encontrar los determinantes de las capacidades de innovación en las organizaciones dedicadas al cuidado de la salud. En primer lugar, se elaboró el modelo estructural y de medida que diera cuenta de las relaciones entre las variables latentes y las manifiestas. Como segundo aspecto se identificó el modelo. Posteriormente se procedió a calcular los índices de ajuste y mediante algunas técnicas de métodos multivariados; se analizaron las áreas del mismo que no se ajustaban adecuadamente para intervenirlas y así conseguir un óptimo ajuste. También se plantearon algunas hipótesis que nos permitieran probar las relaciones de causalidad positiva entre las Capacidades de Innovación y las variables. Respecto a la muestra, se logró tener un total de 283 observaciones extraídas de la red de hospitales de alta complejidad en Colombia durante los años 2016 y 2017. Se obtuvo un modelo con un ajuste significativo y que diera cuenta de los detonantes de las Capacidades de Innovación en las organizaciones de salud. Finalmente se demostró la incidencia positiva que tiene la gestión del conocimiento, del talento humano, las capacidades directivas, la cultura organizacional y algunos factores asociados a la organización de salud sobre dichas capacidades.
\end{abstract}

Palabras clave: capacidades, capacidades de innovación, gestión del conocimiento, cultura organizacional, organizaciones de salud, talento humano, SEM.

Clasificación JEL: I10; M10; 030.

MSC2010: 62F03; 62H15; 62H25; 62J10; 92C50. 


\title{
Innovation capabilities in health organizations. Proposal of a model through structural equation models
}

\begin{abstract}
In the present work, we try to find the determinants of innovation capabilities in the health care organizations. Firstly, the structural and measurement model was elaborated to account the relations between the latent variables and the manifest ones. Secondly, the model was identified; subsequent to this, we proceeded to calculate the adjustment indexes and using some techniques of multivariate methods. Then, we analyzed the areas of the model that did not fit properly to intervene them and thus achieve an optimal adjustment. We proposed some hypothesis that allowed us to prove positive causal relationships between the Innovation Capabilities and the variables. We had 283 observations extracted from the network of high complexity hospitals in Colombia during the years 2016 and 2017. A model with a significant adjustment that would account for the triggers of Innovation Capabilities in health care organizations was obtained. Finally, the positive impact of knowledge management, human resource management, management capabilities, organizational culture, and some factors associated with the health care Organizations on Innovation Capabilities was demonstrated.
\end{abstract}

Keywords: capabilities, innovation capabilities, knowledge management, organizational culture, health care organizations, HRM, SEM.

JEL classification: I10; M10; 030.

MSC2010: 62F03; 62H15; 62H25; 62J10; 92C50.

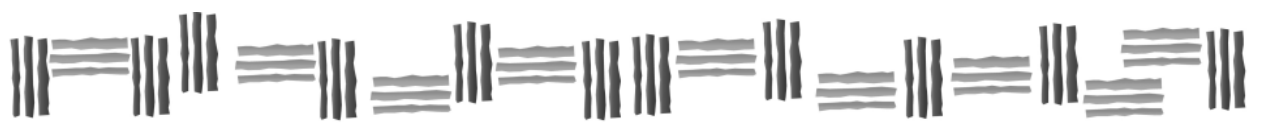




\section{Introducción.}

Actualmente las organizaciones en salud están fuertemente influenciadas por el llamamiento que se hace desde la sociedad para que, por un lado, sean más competitivas, mientras se genera valor para sus pacientes. Es así como, por ejemplo, un foro sobre imperativos en la innovación en salud liderado por la Escuela de Harvard, declara que es necesario reducir costos mientras se incrementa la calidad; además resaltan la importancia de trasladar las capacidades generadas en la creación de nuevos productos a la mejora de procesos. De igual manera es de vital importancia cambiar el paradigma del éxito basado en el volumen de sus operaciones, por unos resultados basados en la generación de valor para sus usuarios que: redunde en vidas salvadas, mejore la salud en los pacientes, prolongue los años de vida saludables (Chin et al., 2012).

En igual sentido resaltan algunas tendencias que moldean y constriñen las acciones de las organizaciones dedicadas al cuidado de la salud. Por ejemplo, la liberación de los mercados ha permitido ofertar servicios de salud a unos costos menores; a su vez también ha generado enormes oportunidades a las instituciones que brindan servicios especializados a unos costos muy competitivos. También se presenta un fenómeno asociado con dichas tendencias y gracias a las TICs que permiten reducir constantemente la brecha en lo que a asimetrías de información en la relación médico paciente se refiere. También es notorio un cambio de la gestión de salud: donde antes se centraba en el hospital y hoy se centra en el paciente. Se imponen nuevos modelos de gestión en salud que empoderan a los pacientes para que se comprometan con su autocuidado. Todo lo anteriormente planteado presiona fuertemente a las organizaciones de salud para que brinden respuestas a los retos y desafíos que hoy les presenta la sociedad. Precisamente para darle respuesta a los desafíos previamente mencionados, surgen las Capacidades de Innovación, en adelante IC (por sus siglas en inglés). La esencia de las IC parte de una solución de problemas y evolucionan en el tiempo a través de un proceso de aprendizaje colectivo y representan una forma superior y distintiva de combinar, asignar recursos que permitan generar nuevos productos, servicios y modelos de negocios que generen valor para los pacientes.

Dada la lógica anteriormente expuesta, surge una problemática a la que se desea dar respuesta que se hace plausible mediante la siguiente cuestión: ¿Es posible plantear y estimar un modelo que dé cuenta de los diversos detonantes y brinden respuestas a las múltiples relaciones que inciden sobre dichas IC?

\section{Marco Teórico.}

La forma más expedita de lograr la anhelada ventaja competitiva es a través de la generación de las Capacidades de Innovación por parte de las organizaciones. Para Lawson y Samson (2001) dichas capacidades pueden definirse como cierta habilidad de la empresa para continuamente transformar ideas y conocimiento en nuevos productos, procesos y sistemas para el beneficio de la misma. La importancia de las IC, está centrada en la generación de valor en un entorno de alta competencia, el cambio acelerado de la tecnología y continuos cambios en los gustos y preferencia de los consumidores que han llevado a las empresas a encontrar ventajas competitivas que le permiten crecer en el mercado (Lawson \& Samson, 2001; Pen, Scroeder \& Shah, 2008; Black \& Synam ,1997). Por tanto, hoy en día las CI se consideran como un mecanismo importante para ser más competitivos y para sobrevivir en el mundo de los negocios globales (Salaman \& Storey, 2002). La innovación brinda a las firmas varias ventajas estratégicas, tales como: la disminución de costos, la diferenciación a través de desarrollo de nuevos productos y servicios y el aumento de la calidad; de no haber innovación, entonces no se puede hablar de crecimiento y competitividad (Yesil et al., 2013; Salaman \& Storey, 2002).

De la revisión de literatura acerca de los modelos asociados con las IC se puede concluir que están asociados con una gran diversidad de factores internos y externos. En una primera fase de la presente investigación se encontraron la diversidad de factores que guardan alguna relación con las CI (Villegas, Montes \& López, 2016). En una investigación posterior se encontraron nueve modelos para el sector salud, como se puede apreciar en la Tabla 1. Vale la pena aclarar que el encuadre conceptual 
se da gracias a la riqueza teórica brindada por dicha corriente y al conjunto de determinantes asociados para generar IC.

Tabla 1. Modelos de Innovación en organizaciones de salud.

\begin{tabular}{|c|c|c|}
\hline Autor(es) & Determinantes de las IC & Principales contribuciones del modelo \\
\hline $\begin{array}{c}\text { (Kaluzny, } \\
1974)\end{array}$ & $\begin{array}{l}\text { Tecnología, estructura organizacional, } \\
\text { habilidades, actitud de los } \\
\text { colaboradores frente a la innovación, } \\
\text { nivel de educación, capacidades } \\
\text { directivas, valores, baja formalización, } \\
\text { descentralización en la toma de } \\
\text { decisiones, estilos de liderazgo, } \\
\text { estructura de los canales de } \\
\text { comunicación, tamaño de la } \\
\text { organización, recursos disponibles y } \\
\text { grado de colaboración con otras } \\
\text { organizaciones de salud. }\end{array}$ & $\begin{array}{l}\text { El autor propone un modelo basado en lo siguiente: } \\
\text { 1) Tanto para el médico como el adoptante de la } \\
\text { innovación, se presentan tres conjuntos de variables: } \\
\text { variables sociodemográficas y de personalidad del } \\
\text { médico, su estatus sociométrico y características de } \\
\text { su práctica médica; 2) Las variables asociadas con la } \\
\text { innovación organizacional, son clasificadas como: } \\
\text { características de los individuos dentro de la } \\
\text { organización, la estructura y procesos de control } \\
\text { organizacional, y 3) el contexto o ambiente en el que } \\
\text { se mueve la entidad. }\end{array}$ \\
\hline $\begin{array}{l}\text { (Kimberly \& } \\
\text { Evanisko, } \\
\text { 1981) }\end{array}$ & $\begin{array}{l}\text { Liderazgo, seguridad en el cargo, } \\
\text { trayectoria del individuo, nivel } \\
\text { educativo, naturaleza de la participación } \\
\text { de los líderes en la organización, } \\
\text { receptividad a la innovación, } \\
\text { centralización, especialización, tamaño, } \\
\text { diferenciación funcional, integración } \\
\text { externa, competencia, tamaño de la } \\
\text { ciudad y del hospital. }\end{array}$ & $\begin{array}{l}\text { El modelo propuesto tiene tres focos sobre los que se } \\
\text { centra la innovación: características individuales, del } \\
\text { ambiente y el organizacional, que son determinantes } \\
\text { en el mismo. }\end{array}$ \\
\hline $\begin{array}{c}\text { (Plsek, } \\
2003)\end{array}$ & $\begin{array}{l}\text { Ideas innovadoras, liderazgo, contexto } \\
\text { receptivo para el cambio, conocimiento, } \\
\text { habilidades y tecnología. }\end{array}$ & $\begin{array}{l}\text { El modelo conceptualiza tres elementos } \\
\text { interrelacionados, como el proceso de generación, } \\
\text { implementación y adopción generalizada de ideas de } \\
\text { innovación, a través de sus procesos, estructuras y } \\
\text { patrones. Dichos elementos están embebidos en un } \\
\text { sistema societal más amplio, político e industrial, } \\
\text { influenciados por el conocimiento y la naturaleza de } \\
\text { las fuerzas de influencia. }\end{array}$ \\
\hline $\begin{array}{l}\text { (Fleuren et } \\
\text { al., 2004) }\end{array}$ & $\begin{array}{l}\text { Rotación de personal, conocimiento del } \\
\text { personal, habilidades y apoyo percibido } \\
\text { de colegas, estrategia, tamaño, } \\
\text { estructura, procesos participativos, } \\
\text { relación con otros departamentos u } \\
\text { organizaciones, grado de capacidad del } \\
\text { personal en la organización o } \\
\text { departamento que implementa la } \\
\text { innovación, número de usuarios } \\
\text { potenciales ser alcanzado. }\end{array}$ & $\begin{array}{l}\text { Propusieron cuatro tipos de determinantes: } \\
\text { Contextual, organizacional, características de la } \\
\text { persona y características de la estrategia de } \\
\text { innovación. También afirman que los determinantes } \\
\text { juegan un papel importante en la estrategia y en cada } \\
\text { fase de la difusión de la innovación. Relacionaron un } \\
\text { conjunto de determinantes que pueden obstaculizar } \\
\text { el proceso de innovación. }\end{array}$ \\
\hline $\begin{array}{r}(\mathrm{Dje} \\
200\end{array}$ & $\begin{array}{l}\text { Habilidad, aprendizaje, competencias } \\
\text { (competencias } \\
\text { intraorganizacionales } \\
\text { interorganizacionales), tecnología. }\end{array}$ & $\begin{array}{l}\text { El modelo propuesto es un servicio constituyente } \\
\text { denominado concentrador o HUB (por su } \\
\text { significado en inglés), lo cual es una combinación de } \\
\text { servicios básicos y periféricos. El modelo se ve } \\
\text { como una función de producción } H=g(\mathrm{~m}) \text {, en la } \\
\text { que el } H \text { denota el producto "salud" y } m \text { la "atención } \\
\text { médica". }\end{array}$ \\
\hline $\begin{array}{l}\text { (Parnaby \& } \\
\text { Towill, } \\
\text { 2008) }\end{array}$ & $\begin{array}{l}\text { Aprendizaje, tecnología, trabajo en } \\
\text { equipo, equipo de proceso (enfermeras, } \\
\text { médicos, recepcionista), recursos, } \\
\text { cultura, liderazgo, motivación, } \\
\text { comunicación. }\end{array}$ & $\begin{array}{l}\text { Propone un modelo en cuatro fases: realización, } \\
\text { investigación, exploración y evolución. La salida es } \\
\text { la entrega de un sistema de innovación en un proceso } \\
\text { de mejora continua gracias al autoaprendizaje. }\end{array}$ \\
\hline & $\begin{array}{l}\text { Tecnología de la información, } \\
\text { necesidades de los pacientes, cultura }\end{array}$ & $\begin{array}{l}\text { Los autores proponen un modelo conceptual } \\
\text { centrado en el paciente y sirviendo a seis propósitos }\end{array}$ \\
\hline
\end{tabular}




\begin{tabular}{|c|c|c|}
\hline $\begin{array}{l}\text { (Omachunu } \\
\quad \& \\
\text { Einspruch, } \\
2010)\end{array}$ & $\begin{array}{l}\text { organizacional, colaboración y trabajo } \\
\text { en equipo, liderazgo y manejo de los } \\
\text { costos. }\end{array}$ & $\begin{array}{l}\text { distintos (tratamiento, diagnóstico, prevención, } \\
\text { educación, investigación y divulgación). Al servir a } \\
\text { estos propósitos, la organización de salud debe } \\
\text { gestionar eficazmente la calidad, los costos, la } \\
\text { seguridad, la eficiencia y los resultados. }\end{array}$ \\
\hline $\begin{array}{c}\text { (Iestyn, } \\
2011 \text { ) }\end{array}$ & $\begin{array}{l}\text { Estructura, niveles de recursos, cultura } \\
\text { organizacional, diferenciación y } \\
\text { especialización, empoderamiento, } \\
\text { conformación de redes, toma de riesgos, } \\
\text { incentivos y recompensa, clima } \\
\text { organizacional, capacidad de absorción, } \\
\text { liderazgo, recursos (financieros y } \\
\text { humanos); las actitudes y experiencias } \\
\text { de los grupos profesionales, canales } \\
\text { actuales y niveles de intercambio de } \\
\text { información, gestión del conocimiento, } \\
\text { mejores prácticas, sistemas de } \\
\text { información y herramientas para la } \\
\text { toma de decisiones, desarrollo de } \\
\text { habilidades, la creación de sentido, la } \\
\text { narración de historias y la } \\
\text { infraestructura. }\end{array}$ & $\begin{array}{l}\text { El autor propone un modelo multideterminante y } \\
\text { multicapa con tres niveles: contextual, interno e } \\
\text { interorganizacional con estrecha relación entre ellos. } \\
\text { En los factores internos, existen tres elementos } \\
\text { básicos: cultura organizacional, estructura y niveles } \\
\text { de recursos. El autor resalta el papel del desarrollo } \\
\text { de incentivos para apoyar la adopción de nuevas } \\
\text { ideas y servicios además de la gestión del } \\
\text { conocimiento. Todo lo anterior es importante para } \\
\text { fortalecer las capacidades. }\end{array}$ \\
\hline $\begin{array}{l}\text { (Jacobs et } \\
\text { al., 2014) }\end{array}$ & $\begin{array}{l}\text { Tamaño de la organización estructura, } \\
\text { madurez de la misma, papel de la } \\
\text { implementación de políticas y prácticas } \\
\text { de la organización (IPP), estrategia, } \\
\text { recompensas, percepción del clima, } \\
\text { edad de la organización de salud, años } \\
\text { de experiencia, especialidad, } \\
\text { capacitación y habilidades. }\end{array}$ & $\begin{array}{l}\text { Los autores proponen tres grupos como } \\
\text { determinantes de la innovación: a) relacionados con } \\
\text { la organización, b) con los médicos y c) relacionados } \\
\text { con la implementación de políticas y prácticas. }\end{array}$ \\
\hline
\end{tabular}
Fuente: Adaptado de (López, Villegas \& Rodríguez, 2017).

\section{Metodología.}

\subsection{Muestra.}

Para el cálculo de la muestra usamos la metodología propuesta por Marsh et al. (1998), que mediante simulación de Monte Carlo encontró la ecuación 1 que permite el cálculo de una muestra en el análisis SEM:

$$
\begin{aligned}
& \quad \text { Ecuación 1: } N_{1} \geq 50 r^{2}-450 r+1100, \operatorname{con} r=\frac{\sum_{1}^{C} \xi_{i}}{C} \\
& \text { donde: } \boldsymbol{N}_{\mathbf{1}}=\text { muestra mínima } \\
& \xi_{\boldsymbol{i}}=\text { número de variables manifiestas } X_{i} \text { por cada constructo y } \\
& \boldsymbol{C}=\text { número de variables latentes empleados en el modelo }
\end{aligned}
$$

\section{Con $\boldsymbol{i}$ variando desde 1 hasta $C$}

Basándonos en la información del modelo planteado en la Figura 1 y reemplazando con la información suministrada por el mismo, se pudo obtener el tamaño muestral, tal y como se puede apreciar en la Tabla 2. 
Figura 1. Modelo de medición y estructural de los predictores de las Capacidades de Innovación en las Organizaciones de Salud.

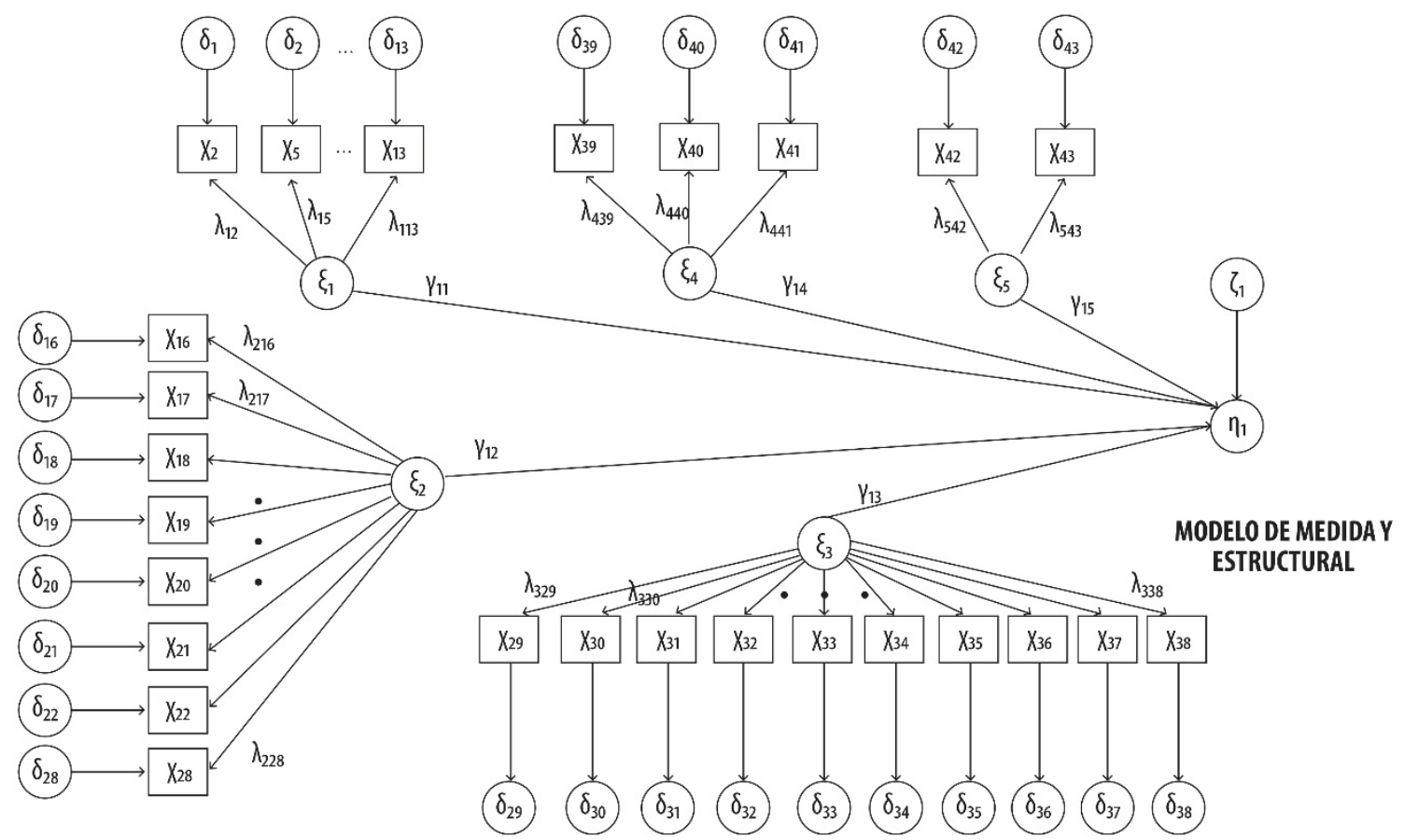

Fuente: Elaboración propia.

Tabla 2. Tamaño muestral.

\begin{tabular}{|c|l|}
\hline Característica & \multicolumn{1}{|c|}{ Valor } \\
\hline Variables latentes & 6 \\
\hline Promedio de las variables manifiestas & $(15+7+9+3+2) / 6$ \\
\hline Tamaño mínimo de la muestra: $N_{1} \geq$ & 200 \\
\hline
\end{tabular}

Fuente: Elaboración propia.

La metodología sugerida por Marsh et al. (1998) indica una muestra mínima de 200. Al respecto Hoelter (1983) ratifica dicho hallazgo. Sin embargo, se pudo obtener una muestra bastante representativa de 283 observaciones, las mismas se obtuvieron de la red de hospitales de alta complejidad en Colombia entre los años 2016 y 2017. Dicha muestra incluyó a médicos (27.9\%), auxiliares $(22.6 \%)$, personal de enfermería (25.8\%) y administrativos (23.7\%); siempre respetando que las muestras fuesen homogéneas y aleatorias para cada hospital con edades desde los 20 hasta los 70 años, con media de 35 años. Respecto al nivel de estudio, el $1.1 \%$ tiene doctorado, el $25.4 \%$ especialización, el $6.7 \%$ maestría, el $42.8 \%$ pregrado y sin título académico el $1.4 \%$.

\subsection{Instrumento.}

Según revisión sistemática de literatura (Villegas, Montes \& López, 2016) y reuniendo los trabajos expuestos en la Tabla 1, donde se llegó en total a 539 trabajos analizados, no se pudo evidenciar la existencia de trabajos en la dirección de la presente investigación, y mucho menos con un instrumento que analice los predictores de las IC en las organizaciones dedicadas a la salud de las personas. Quizás el trabajo que más se acerca es el propuesto por Fierro, Cantú, Martínez y López (2016). Por todo lo anterior fue necesario la elaboración de un instrumento totalmente nuevo. Finalmente, los encuestados calificaron con escala métrica de uno a cinco acerca de la gestión de la empresa de salud en lo relativo a cada uno de los predictores de las capacidades de innovación, de manera que calificaban con 1 en el caso de que no se haya realizado ninguna actividad tendente a mejorar la característica indagada y el 
máximo con un valor de 5, para representar que se han obtenido resultados positivos frente al aspecto que se preguntó.

\subsection{Técnica aplicada.}

El Modelamiento con Ecuaciones Estructurales (SEM) es una herramienta estadística de análisis multivariante que tiene un gran poder explicativo; además se resalta su poder de análisis de relaciones múltiples de dependencia cruzada, y el poder incorporar variables latentes. SEM prueba modelos teóricos utilizando el método científico de pruebas de hipótesis para avanzar en nuestra comprensión de las complejas relaciones entre constructos.

Modelo Estructural y Modelo de Medida. El primero tiene la función obtener los pesos de las relaciones para comparar las relaciones causales, así como también la influencia directa e indirectas entre las variables. Por el contario, el segundo, muestra la incidencia de cada una las variables manifiestas sobre las observables. En la Figura 1 se pueden observar ambos modelos. Ahora para su modelación y mejor entendimiento es necesario plantear las siguientes ecuaciones matriciales:

1) Modelo de medición: $X=\Lambda_{x} \xi+\delta$ con

$\mathrm{X}_{(q X 1)}=$ variables manifiestas (35)

$\Lambda_{x(q \times s)}=$ cargas asociadas a las variables manifiestas (35X5)

$\xi_{(s x 1)}=$ vector de latentes (5)

$\delta_{(q \times 1)}=$ errores de medición de las latentes (35)

2) Modelo estructural: $\eta=\Gamma \xi+Z$ con

$\eta_{r \times 1}=$ Matriz con las cargas de los Factores dependientes (1).

$\Gamma_{r x s}=$ Matriz con los efectos directos de $\xi$ sobre $\eta(5)$

$\xi_{s x 1}=$ Contiene una matriz con los factores independientes (5)

\subsection{Hipótesis de trabajo.}

Para la presente propuesta se toman las hipótesis de tercer grado, como primera instancia por la multiplicidad de variables asociadas a la CI y como segunda, este tipo de hipótesis establecen relaciones entre variables complejas, que están constituidas a su vez por sistemas de variables.

Tabla 3. Hipótesis de trabajo.

\begin{tabular}{|c|c|c|c|}
\hline Hipótesis Nula & Parámetro & Hipótesis Alternativa & Parámetro \\
\hline $\mathbf{H}_{10} \mathbf{0}$ & $\gamma_{11}=0$ & $\mathbf{H}_{12}$ & $\gamma_{11} \neq 0$ \\
\hline $\mathbf{H}_{20}$ & $\gamma_{12}=0$ & $\mathbf{H}_{2} \mathbf{a}$ & $\gamma_{12} \neq 0$ \\
\hline $\mathbf{H}_{30}$ & $\gamma_{13}=0$ & $\mathbf{H}_{3} \mathbf{a}$ & $\gamma_{13} \neq 0$ \\
\hline $\mathbf{H}_{40}$ & $\gamma_{14}=0$ & $\mathbf{H}_{4} \mathbf{a}$ & $\gamma_{14} \neq 0$ \\
\hline $\mathbf{H}_{50}$ & $\gamma_{15}=0$ & $\mathbf{H}_{5} \mathbf{a}$ & $\gamma_{15} \neq 0$ \\
\hline
\end{tabular}

Fuente: Elaboración propia. 
El propósito de las mismas es probar la incidencia que tiene cada una de las variables latentes frente a las IC como se puede apreciar en la Tabla 4.

Tabla 4. Variables latentes y manifiestas asociadas a las Capacidades de Innovación.

\begin{tabular}{|c|c|c|}
\hline Variables Manifiestas & Variables latentes & Definición \\
\hline $\begin{array}{l}X_{1}, X_{2}, X_{3} X_{4}, X_{5}, X_{6}, X_{7} \\
X_{8}, X_{9}, X_{10}, X_{11}, X_{12}, X_{13} \\
\quad X_{14} y X_{15}\end{array}$ & $\begin{array}{l}\xi_{1}=\text { Gestión del } \\
\text { Conocimiento }(\mathrm{GC})\end{array}$ & $\begin{array}{l}\text { Total de acciones destinadas a almacenar crear, y } \\
\text { reutilizar los conocimientos que permitan el manejo de } \\
\text { las CI. (Börjesson \& Elmquist, 2011; Nonaka \& } \\
\text { Takeuchi, 1995; Cohen \& Levinthal,1990). }\end{array}$ \\
\hline $\begin{array}{l}X_{16}, X_{17}, \quad X_{18} \\
X_{19}, X_{20} X_{21} \text { y } X_{25}\end{array}$ & $\begin{array}{l}\xi_{2}=\text { Capacidades } \\
\text { Directivas }(C D)\end{array}$ & $\begin{array}{l}\text { Habilidad de la empresa para gestionar los recursos } \\
\text { disponibles en la misma con el propósito de conseguir } \\
\text { los resultados planeados (Schreyögg \& Kliesch-Eberl, } \\
\text { 2007; Bravo-Ibarra \& Herrera,2009). }\end{array}$ \\
\hline $\begin{array}{l}X_{29}, X_{30}, X_{31}, X_{33} \\
X_{34}, X_{35}, X_{36}, X_{37} \mathrm{y} \\
X_{38}\end{array}$ & $\begin{array}{l}\xi_{3}=\text { Administración } \\
\text { del Recurso Humano } \\
(\mathrm{GTH})\end{array}$ & $\begin{array}{l}\text { Es una actividad estratégica diseñada con el propósito de } \\
\text { obtener, formar, motivar, retribuir y desarrollar a los } \\
\text { miembros de la organización para que de manera } \\
\text { mancomunada se logren las metas organizacionales. } \\
\text { (Martinez-Roman, Gamero \& Tamayo, 2011). }\end{array}$ \\
\hline$X_{39}, X_{40}$ y $X_{41}$ & $\begin{array}{l}\xi_{4}=\text { Factores } \\
\text { asociados a la } \\
\text { Organización } \\
\text { dedicada al cuidado } \\
\text { de la salud (FAIPS) }\end{array}$ & $\begin{array}{l}\text { Son fortalezas destinadas a aprovechar las oportunidades } \\
\text { de su entorno y a la posibilidad de abordar trabajos } \\
\text { colaborativos con otras organizaciones de salud (Iestyn, } \\
\text { 2011; Jacobs et al., 2014). }\end{array}$ \\
\hline$X_{42}$ y $X_{43}$ & $\begin{array}{l}\xi_{5}=\text { Aspectos } \\
\text { relacionados con la } \\
\text { cultura }(\mathrm{FC})\end{array}$ & $\begin{array}{l}\text { Son elementos de la cultura organizacional tales como } \\
\text { los principios y valores que permite impulsar la } \\
\text { innovación (Naranjo, Jimenez \& Sanz-Valle, 2015). }\end{array}$ \\
\hline
\end{tabular}

Fuente: Elaboración propia.

\subsection{Evaluación del modelo.}

Para lograr el objetivo planteado, en lo relativo a la estimación de los parámetros del modelo será necesario seguir los siguientes procedimientos: 1) Especificar el modelo, 2) Identificar el modelo, 3) Estimar el modelo y 4) Reespecificación del modelo de tal manera que se optimicen sus índices de ajuste y probar finalmente las hipótesis de trabajo.

\section{Resultados.}

\subsection{Especificación del modelo.}

Se elaboró partiendo de un trabajo previo, donde se realizó una revisión sistemática de literatura acerca de los predictores de la capacidad de innovación (Villegas, Montes \& López, 2016), y gracias a un trabajo posterior donde se propuso una ampliación de los mismos aplicados solo y exclusivamente a las organizaciones de salud (ver tabla 1). Dicha especificación se puede apreciar en la Figura 1.

\subsection{Identificación del modelo.}

La modelización elaborada mediante SEM se representa mediante un gráfico orientado donde se observan las relaciones entre las variables manifiestas y una serie de parámetros o pesos de cada una de las aristas. Según Vasquez (2013) dicha relación puede ser expresada por la siguiente ecuación: $M=$ $\left\{G, \theta_{G}\right\}$, dónde: $G=$ gráfico orientado, $\theta_{G}=$ parámetros y $\mathrm{M}=$ Modelo de Ecuaciones Estructurales. El modelador construye $G$ y desea obtener $\theta_{G}$ a partir de la muestra, además de los índices que indiquen si $G$ se ajusta.

Existe una regla para identificar el modelo, que corresponde a la condición de orden bajo la que el número de parámetros libres a ser estimados deben ser menores que los distintos valores en la matriz 
$\mathrm{S}$, que se calcula mediante la siguiente formula: $\mathrm{p}(\mathrm{p}+1) / 2$, donde $\mathrm{p}$ son las variables manifiestas. Sumado a esto y basado en los grados de libertad (gl), se sabe ciertamente cuál es la identificación del modelo. Entonces $\mathrm{gl}=[\mathrm{p}(\mathrm{p}+1) / 2-$ número de parámetros a estimar $]$. Cabe anotar que el modelo está identificado si todos los parámetros lo están, es decir, si existe una solución única para cada uno de los parámetros estimados. Por todo lo anterior y de acuerdo a la Figura 1 y la Tabla 5 se pueden observar los resultados para la identificación del modelo, gracias al cálculo de los grados de libertad del mismo.

Tabla 5. Cálculo de la identificación del modelo.

\begin{tabular}{|l|r|}
\hline \multicolumn{1}{|c|}{ Indicador para el cálculo } & \multicolumn{1}{c|}{ Valor } \\
\hline Variables observables & 35 \\
\hline Varianzas de las variables observables & 35 \\
\hline Varianza de las variables latentes & 5 \\
\hline Número de paths o senderos & 35 \\
\hline Número de correlaciones & 0 \\
\hline Errores de las covarianzas & 0 \\
\hline Grados de libertad $(g l=p(p+1) / 2$ - número de parámetros a estimar $)$ & $\mathbf{5 8 9}$ \\
\hline
\end{tabular}

Fuente: Elaboración propia.

\subsection{Estimación del modelo.}

Basados en el modelo planteado en la Figura 1 y después de haber introducido el código en el programa estadístico $\mathrm{R}$ versión 3.4.0 y especialmente con la ayuda de la librería lavaan, se pudo obtener la estimación del mismo utilizando el método de Máxima Verosimilitud o ML (Maximum Likelihood), que es muy robusto en el cálculo de los parámetros cuando las variables observables son continuas como es el caso objeto de estudio (Tabla 6).

Tabla 6. Índices de ajuste del modelo.

\begin{tabular}{|l|r|r|r|}
\hline \multicolumn{1}{|c|}{ Índice } & \multicolumn{1}{|c|}{ Valor } & \multicolumn{1}{c|}{ Intervalo de Confianza } & \multicolumn{1}{c|}{-value } \\
\hline SRMR & 0.080 & 0.076 hasta 0.085 con un $90 \%$ & Significativa $p=0.000<0.005$ \\
\hline Cmin $\left(X^{2}=1864 / \mathrm{gl}=660\right)$ & 2.9 & NA & NA \\
\hline CFI & 0.867 & NA & NA \\
\hline TLI & 0.859 & NA & NA \\
\hline
\end{tabular}

Fuente: Elaboración propia.

\subsection{Respecificación del modelo y estimación de parámetros para la comprobación de hipótesis.}

Aquí se buscó la modificación del mismo planteado en la Figura 1 con el propósito de encontrar uno más parsimonioso, es decir, que se ajuste con un menor número de parámetros o sea con menos número de relaciones causales, ya sea suprimiendo variables o modificando las relaciones existentes entre los constructos o los predictores. Dicha modificación se hizo utilizando nuevamente R por medio de los índices de modificación y especialmente el análisis de los residuos para los reactivos. Con respecto a la bondad de ajuste del modelo, es necesario aclarar que el mismo se refiere a la exactitud de los supuestos del modelo especificado para determinar si el modelo es correcto y sirve como aproximación al fenómeno real, precisando así su poder de predicción. Los índices del modelo reespecificado se pueden observar en la Tabla 7. El modelo final junto con sus parámetros estimados, se pueden apreciar en la Figura 2 y Tabla 8 respectivamente. 
Tabla 7. Índices de bondad de ajuste del modelo reespecificado.

\begin{tabular}{|c|c|c|}
\hline Tipo & Indicador & Valor \\
\hline \multirow[t]{3}{*}{ Ajuste Absoluto } & $\mathrm{X}^{2}$ & 740.979 \\
\hline & Df & 294 \\
\hline & RMSEA al 90\% de confianza & 0.073 \\
\hline \multirow{2}{*}{$\begin{array}{l}\text { Medidas incrementales de ajuste } \text { o } \\
\text { Comparativos }\end{array}$} & CFI & 0.924 \\
\hline & TLI & 0.916 \\
\hline Ajuste de Parsimonia & Cmin & 2.52 \\
\hline
\end{tabular}

Fuente: Elaboración propia

Tabla 8. Valores estimados de los parámetros del modelo reespecificado.

\begin{tabular}{|c|c|c|c|c|c|c|c|}
\hline Parámetro & Estimado & $\begin{array}{c}\text { Std- } \\
\text { error }\end{array}$ & $\begin{array}{r}\text { Critical } \\
\text { Ratio }\end{array}$ & Parámetro & Estimado & $\begin{array}{r}\text { Std- } \\
\text { error }\end{array}$ & $\begin{array}{c}\text { Critical } \\
\text { Ratio }\end{array}$ \\
\hline$\xi_{1}=\sim \eta_{1}$ & 1.584 & 0.151 & 10.496 & & & & \\
\hline$\lambda_{12}$ & 0.529 & 0.047 & 11.237 & $\lambda_{110}$ & 0.359 & 0.035 & 10.370 \\
\hline$\lambda_{15}$ & 0.429 & 0.039 & 10.986 & $\lambda_{111}$ & 0.347 & 0.034 & 10.246 \\
\hline$\lambda_{16}$ & 0.657 & 0.058 & 11.421 & $\lambda_{112}$ & 0.298 & 0.030 & 10.017 \\
\hline$\lambda_{17}$ & 0.458 & 0.042 & 10.813 & $\lambda_{113}$ & 0.437 & 0.040 & 10.799 \\
\hline$\lambda_{19}$ & 0.455 & 0.042 & 10.945 & & & & \\
\hline$\xi_{2}=\sim \eta_{1}$ & 1.987 & 0.221 & 9.011 & & & & \\
\hline$\lambda_{216}$ & 0.414 & 0.040 & 10.433 & $\lambda_{220}$ & 0.323 & 0.034 & 9.381 \\
\hline$\lambda_{218}$ & 0.467 & 0.048 & 9.700 & $\lambda_{225}$ & 0.450 & 0.044 & 10.214 \\
\hline$\lambda_{219}$ & 0.504 & 0.047 & 10.647 & & & & \\
\hline$\xi_{3}=\sim \eta_{1}$ & 1.847 & 0.174 & 10.630 & & & & \\
\hline$\lambda_{330}$ & 0.350 & 0.033 & 10.478 & $\lambda_{336}$ & 0.377 & 0.035 & 10.033 \\
\hline$\lambda_{331}$ & 0.375 & 0.035 & 10.693 & $\lambda_{337}$ & 0.181 & 0.000 & 9.019 \\
\hline$\lambda_{333}$ & 0.529 & 0.047 & 11.147 & $\lambda_{338}$ & 0.235 & 0.024 & 9.893 \\
\hline$\lambda_{335}$ & 0.290 & 0.028 & 10.382 & & & & \\
\hline$\xi_{4}=\sim \eta_{1}$ & 1.600 & 0.164 & 9.733 & & & & \\
\hline$\lambda_{439}$ & 0.478 & 0.049 & 9.843 & $\lambda_{441}$ & 0.174 & 0.032 & 5.427 \\
\hline$\lambda_{440}$ & 0.508 & 0.051 & 10.023 & & & & \\
\hline$\xi_{5}=\sim \eta_{1}$ & 2.395 & 0.305 & 7.854 & & & & \\
\hline$\lambda_{542}$ & 0.148 & 0.027 & 8.312 & $\lambda_{543}$ & 0.148 & 0.027 & 5.496 \\
\hline
\end{tabular}

Fuente: Elaboración propia.

\section{Análisis.}

Respecto a la identificación del modelo, se encontró que estaba sobreidentificado, como se pudo observar en el cálculo de los grados de libertad (Tabla 5). Se obtuvo un valor mayor que cero, lo que concuerda con lo propuesto por Shumaker y Lumax (2010), quienes afirman que existen tres niveles para identificarlos, y que ello depende de la cantidad de información de la matriz $\mathrm{S}$ de varianzascovarianzas necesarias para estimar únicamente los parámetros del modelo. Un modelo es no identificado si uno o más parámetros no pueden ser únicamente determinados porque no hay suficiente información en la matriz $S$. El modelo es solo identificado si todos los parámetros son únicamente determinados debido a la existencia de la información necesaria en la matriz $S$. Es sobre estimado si hay más de una forma de la estimación de los parámetros porque hay mucha información, como es el 
caso que se pudo evidenciar en la Figura 1. Por todo lo anterior, es claro que, con respecto a la estimación de los parámetros del modelo, cuyo objetivo es determinar las cargas de las relaciones entre los constructos y cada uno de los predictores de la capacidad de innovación, queda totalmente demostrado, por la identificación del modelo, la plausibilidad para el cálculo de dichos parámetros.

En lo relativo al ajuste de bondad del modelo planteado en la Figura 1, se puede afirmar lo siguiente:

1) El Cmin como índice de parsimonia, está entre 1 y 3 que es lo ideal (Shumaker \& Lumax,2010).

2) El RMSEA es igual a 0.08 (Browne \& Cudeck, 1993; Hu \& Bentler, 1995), indican que es ajuste aceptable, inclusive el límite superior del intervalo de confianza está ligeramente superior al aceptable. El modelo planteado se puede aceptar porque se acerca a un buen ajuste gracias al Cmin y al RMSEA (ver Tabla 6).

Ahora, si bien los indicadores asociados a la Figura 1 mostraron ajuste aceptable, también es cierto que algunos como el CFI y el TLI no lo hacen adecuadamente. Por lo tanto, es necesario buscar las áreas donde no lo hace, con el propósito de mejorarlo para que se aproxime a un modelo mucho más parsimonioso, y en consecuencia se obtenga un mejor ajuste y pueda resultar totalmente válido el modelo planteado. Gracias al análisis de la información brindada por los índices de modificación se pudo mejorar ostensiblemente la $X^{2}$, como bien se puede apreciar en la Tabla 7. El propósito de los modelos con ecuaciones estructurales radica en determinan la diferencia entre la matriz de covarianza $\Sigma(\theta)$ obtenida basada en el modelo y la matriz de covarianza de la muestra $S$. Por lo tanto, la divergencia entre las dos matrices anteriores aparece en una matriz con los residuos $(\Sigma(\theta)-S)$, hay un residuo para cada pareja de variables utilizadas en el modelo. Para aquellos datos mayores a 1.96 se consideran grandes (Shumaker \& Lumax, 2010) y por ende deben eliminarse dichos predictores. Finalmente, el modelo definitivo se puede apreciar en la Figura 2 y en la Tabla 7 donde se nota claramente la mejora del modelo. Allí se puede observar los siguiente:

1) El Cmin o ajuste de parsimonia sigue en el rango deseado (Shumaker \& Lumax,2010).

2) Tanto el $X^{2}$ como los grados de libertad ( $d f$ ) disminuyeron.

3) El CFI, ahora está por encima de 0.9 que es un buen ajuste (Hu \& Bentler, 1998).

4) Lo mismo sucede con el TLI que también muestra un buen ajuste por encima de 0.9 (Hu \& Bentler, 1998).

Respecto a la prueba de las hipótesis, el análisis de los parámetros $\left(\gamma_{11}, \gamma_{12}, \gamma_{13}, \gamma_{14}\right.$ y $\left.\gamma_{15}\right)$ resultaron significativos, el Critical Radio (CR) es mucho mayor que 1,96. De tal manera que las hipótesis planteadas sobre las incidencias de las latentes exógenas sobre las endógenas tienen sentido, como se puede observar en la Tabla 9 donde se presenta el resumen. En cuanto a los predictores de las capacidades de innovación, se puede observar en la Tabla 8 que resultaron mayores a cero y significativos.

Tabla 9. Comprobación de hipótesis.

\begin{tabular}{|c|c|c|c|c|}
\hline Hipótesis Nula & Parámetro & Hipótesis Alternativa & Parámetro & Resultado \\
\hline $\mathbf{H}_{10} \mathbf{0}$ & $\gamma_{11}=0$ & $\mathbf{H}_{1} \mathbf{a}$ & $\gamma_{11} \geq 0$ & Se rechaza $\mathbf{H}_{\mathbf{1}}$ \\
\hline $\mathbf{H}_{20}$ & $\gamma_{12}=0$ & $\mathbf{H}_{2} \mathbf{a}$ & $\gamma_{12} \geq 0$ & Se rechaza $\mathbf{H}_{20}$ \\
\hline $\mathbf{H}_{30} \mathbf{0}$ & $\gamma_{13}=0$ & $\mathbf{H}_{3 \mathbf{a}}$ & $\gamma_{13} \geq 0$ & Se rechaza $\mathbf{H}_{30}$ \\
\hline $\mathbf{H}_{40}$ & $\gamma_{14}=0$ & $\mathbf{H}_{4} \mathbf{a}$ & $\gamma_{14} \geq 0$ & Se rechaza $\mathbf{H}_{40}$ \\
\hline $\mathbf{H}_{50}$ & $\gamma_{15}=0$ & $\mathbf{H}_{5 \mathbf{a}}$ & $\gamma_{15} \geq 0$ & Se rechaza $\mathbf{H}_{50}$ \\
\hline
\end{tabular}

Fuente: Elaboración propia. 
Figura 2. Modelo de Medida y Estructural reespecificado.

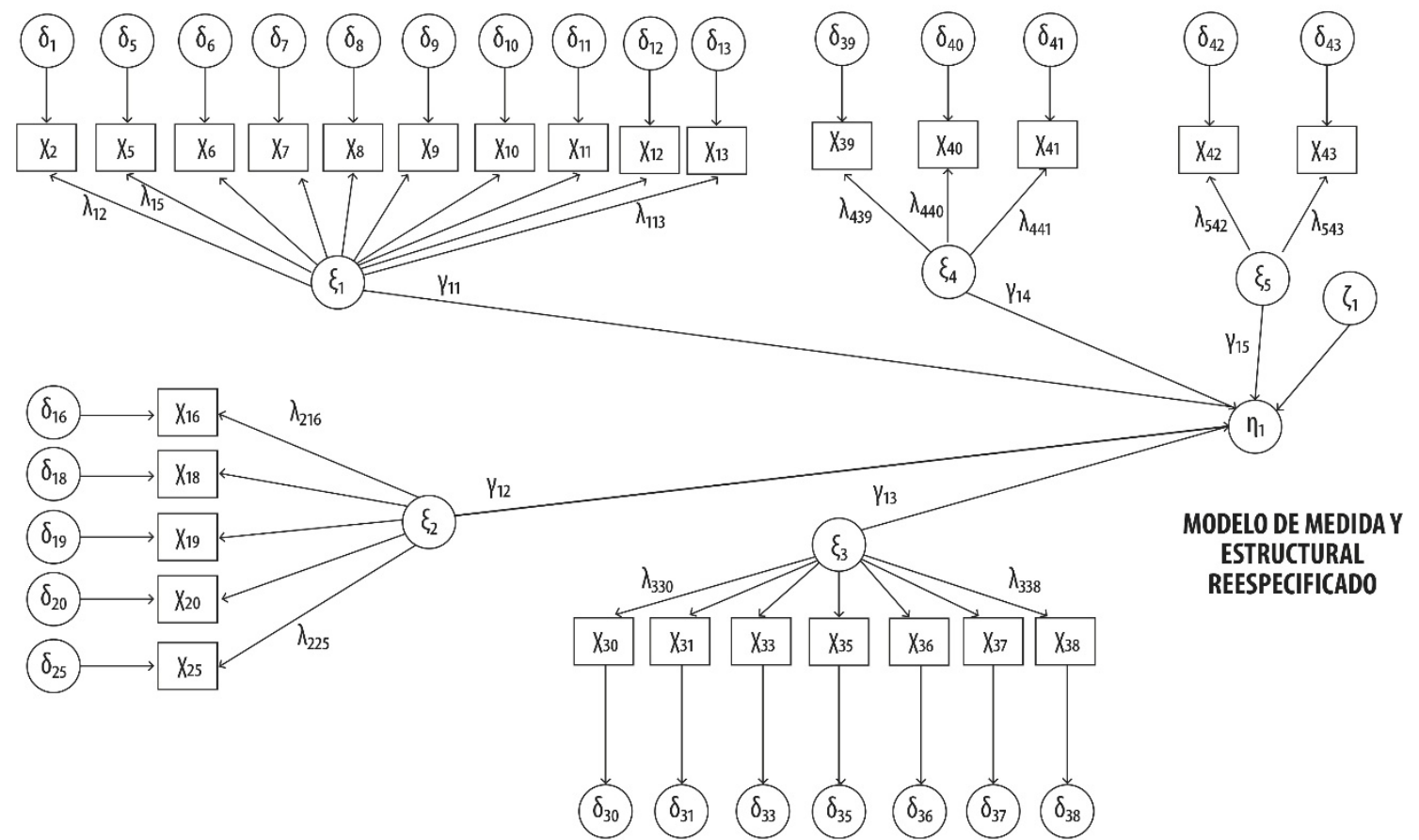

Fuente: Elaboración propia.

\section{Conclusiones.}

La ruta seguida en la metodología nos permite concluir que el modelo planteado en la Figura 1 contiene cinco factores con sus predictores asociados, que después de un primer análisis arroja un ajuste moderado, por un lado, pero por el otro, índices como el CFI y el TLI brindaron luces sobre la necesidad de evaluar el modelo para analizar aquellas áreas donde no ajustaba. Para tal efecto fue necesario reespecificar el modelo con la ayuda de los índices de modificación y con la ayuda del análisis de los residuos estandarizados que también permitieron encontrar problemas de multicolinealidad. Ahora tal y como se pudo observar en la Tabla 7, los índices incrementales aumentaron su valor por encima de 0.9 , sumados con los otros indicadores de ajuste absolutos y de parsimonia indican en su conjunto un buen ajuste, lo que indica que finalmente el modelo se puede aceptar.

Como se puede apreciar en la Figura 2 y la Tabla 8, los factores detonantes de las CI en salud están conformadas por la gestión del conocimiento, las capacidades directivas, la gestión del talento humano, aquellos factores asociados con la organización en salud y, finalmente, los asociados con la cultura organizacional. A continuación, se describen los inductores que resultaron significativos para el modelo:

1) Respecto a la gestión del conocimiento, se hace necesario generar oportunidades de aprendizaje en los colaboradores en lo referente a: resolución de problemas y la búsqueda de alternativas que le permitan dar soluciones efectivas; en la retroalimentación de sus prácticas cotidianas; de igual manera es necesario que las organizaciones de salud transfieran las mejores prácticas en lo que a procesos y/o procedimientos se refiere; al uso de las TIC como instrumento para canalizar ideas, organizar la experiencia y el conocimiento adquirido, en mantener a los miembros cada vez más informados acerca de su labor, para registrar la experiencia adquirida de tal manera que pueda ser compartida en toda la organización; y, por último, se hace necesario mantener una comunicación abierta entre todos sus miembros. 
2) En cuanto a las capacidades directivas, es necesario lograr un consenso sobre la importancia de la innovación para las organizaciones en salud; de igual manera la misma debe contar con líderes que sean los responsables de gestionar los procesos de innovación; además es muy importante que dichos procesos permitan disminuir los costos para dichas organizaciones.

3) En lo relativo a la gestión del talento humano, los predictores que resultaron determinantes están enmarcados en su gran mayoría al desarrollo de habilidades para trabajo en equipo; en la necesidad de motivar a los colaboradores en lo relacionado a la generación de oportunidades de crecimiento profesional; a propiciar el ascenso de los mismos; al enriquecimiento del cargo de forma horizontal y vertical; en valorar las ideas nuevas del personal pues la organización debe estar centrada en el desarrollo de las personas y a promover un ambiente de colaboración entre todos los miembros.

4) En lo relativo a los factores asociados con la organización en salud, es claro que desde ellas se debe promover el trabajo colaborativo con otras organizaciones del sector que permitan trabajar conjuntamente en intereses que las agrupen. Poder compartir procesos y procedimientos que sean reconocidos como susceptibles de ser compartidos y adaptados. Por último, una condición que detecta el estudio es la necesidad de moverse rápidamente para aprovechar oportunidades del entorno.

5) En lo referente a la cultura organizacional, si bien existen factores de la cultura que están entremezclados con los anteriores factores, se pudo evidenciar que los valores cumplen el papel de servir de guía en lo que a innovación se refiere y que además se hace necesario generar un ambiente laboral que propicie los procesos de innovación.

En relación con lo anterior y analizando el poder de cada uno de los factores se puede afirmar que un cambio en una unidad en cualquiera de los siguientes elementos tiene un efecto amplificador sobre las IC, así : a) la gestión del conocimiento afecta en 1.584 ; b) las capacidades directivas tienen un efecto de 1.987 ; c) la gestión del talento humano posee un impacto de 1.847 ; d) los factores asociados con la organización en salud de $1.6 \mathrm{y}$, por último, d) la gestión de la cultura organizacional es la que mayor impacto posee sobre las IC, el efecto total es de 2.395 (Tabla 8).

Ahora, desde una perspectiva teórica, es necesario resaltar que la corriente teórica que trae consigo el abordaje de las IC aplicadas al sector salud está en etapa de introducción y crecimiento. Por lo tanto, el presente modelo es un caldo de cultivo que permite a las organizaciones dedicadas al cuidado salud, el poder desarrollar y consolidar las IC que resultan determinantes en un sector donde es altamente apreciado y reclamado el avance en nuevos productos, o procesos o modelos de negocios, que generen valor, y redunden en una prolongada y mejor calidad de vida de los pacientes.

Finalmente, nos permitió corroborar que los resultados obtenidos guardan concordancia y siguen la línea de los siguientes trabajos relacionados con aquellas organizaciones que están dedicadas al cuidado de la salud -en su gran mayoría de corte descriptivo-: Caccia-Bava, Guimaraes \& Hamminton (2006) resaltan el papel de los aspectos culturales, las Tecnologías de Información y la Comunicación y el conocimiento sobre las CI; esta última relación también es resaltada por Mylopoulos y Scardamalia (2008), Lee y Hong (2014), Ugurluoglu et al. (2013) y Dias y Escoval, 2015. Por su parte, Jaramillo et. al (2008) y Sharma, Conduit y Hill (2014) afirman que el desarrollo y consolidación de IC se hace posible gracias a un conjunto de capacidades, tales como: las directivas, las asociadas con la gestión del conocimiento, las tecnológica, las de aprendizaje o de absorción. Además, las organizaciones en salud deben gestionar los procesos, los contextos organizacionales, el componente humano, la cultura, las alianzas estratégicas y la capacidad de los individuos para integrar y generar ideas. Yan (2014) afirma que la gestión del conocimiento, el capital relacional y la presión del entorno resultan determinantes en la generación de las IC. Thakur, Hsu y Fontenot (2012) encuentran en su trabajo un conjunto de variables que afectan la capacidad para innovar: la cultura organizacional, relación con otras 
organizaciones, la orientación al mercado, usos de las TIC, capacidades directivas, entre otras. Todo lo anterior nos permite generar las bases para estudios posteriores que prosigan en la senda de la consolidación y desarrollo de las IC en el sector salud.

\section{Agradecimientos}

Damos nuestro más sincero agradecimiento por todo su valioso apoyo a los hospitales de la red de alta complejidad en Colombia que participaron en el estudio, sin ellos no hubiese sido posible la presente investigación. Se desea hacer mención especial a las siguientes instituciones: Clínica las Américas, Hospital General de Medellín, E.S.E Hospital Universitario San Jorge, San Vicente Fundación, IPS Universitaria y Clínica Soma.

\section{Referencias}

Black, D.H., \& Synan, C.D. (1997). The learning organization: The sixth discipline? Management Accounting British, 75(10), 70-72.

Börjesson, S., \& Elmquist, M. (2011). Developing Innovation Capabilities: A Longitudinal Study of a Project at Volvo Cars. Creativity and Innovation Management, 20(3), 171-184.

Bravo-Ibarra, E.R., \& Herrera, L. (2009). Capacidad de innovación y configuración de recursos organizativos. Intangible Capital, 5(3), 301-320.

Browne, M.W., \& Cudeck, R. (1993). Alternative ways of assessing model fit. In K.A. Bollen \& J. S. Long. Testing structural equation models. Newsbury: Sage.

Caccia-Bava, M.C., Guimaraes, T. \& Harrington, S.J. (2006). Hospital organization culture, capacity to innovate and success in technology adoption. Journal of Health Organization and Management, 20(3), 194-217.

Chin, W., Hamermesh, R., Huckman, R., McNeil, B., \& Newhouse, J. (2012). 5 Imperatives addressing healthcare's innovation challenge. Forum on Health Care Innovation. Cambridge.

Cohen, W. M., \& Levinthal, D. A. (1990). Absorptive-Capacity - A New Perspective on Learning and Innovation. Administrative Science Quarterly, 35(1), 128-152.

Dias, C., \& Escoval, A., (2015). Hospitals as learning organisations: fostering innovation through interactive learning. Quality Management Health Care, 24(1), 52-59.

Djellal, F. (2005). Mapping innovation dynamics in hospitals. Research Policy, 34, 817-835.

Fierro, E., Cantú, J.L., Martínez, J. \& López O.H. (2016). Predictores de la innovación administrativa: funciones y métodos organizacionales - Hospitales de México y Colombia. $R G B N, 17(54), 806-$ 821.

Fleuren, M., Wiefferink, K., \& Paulussen, T. (2004). Determinants of innovation within health care organizations. International Journal for Quality in Health Care, 16(2), 107-123.

Hoelter, J.W. (1983). The analysis of covariance structures: Goodness-of-fit indices. Sociological Methods and Research, 11, 325-344.

Hu, L., \& Bentler, P.M. (1995). Evaluating model fit. In R. H. Hoyle. Structural equation modeling: Concepts, issues, and applications. London: Sage. 
Iestyn, W. (2011). Organizational readiness for innovation in health care: some lessons from the recent literature. Health Services Management Research, 24, 213-218.

Jacobs, S., Weiner, B., Reeve, B., Hofmann, D., Christian, M., \& Weinberger, M. (2014). Determining the predictors of innovation implementation in healthcare: a quantitative analysis of implementation effectiveness. BMC Health Services Research, 15(6), 1-13.

Jaramillo, H., Latorre, C., Alban, C., \& Lopera, C. (2008). El hospital como organización de conocimiento y espacio de investigación y de formación. Bogotá: Editorial Universidad del Rosario.

Kaluzny, A.D. (1974) Innovation in Health Services: Theoretical Framework and Review of Research. Health Service Research, 9(2), 101-120.

Kimberly, J.R \& Evanisko M.J. (1981). Organizational innovation: The influence of individual, organizational and contextual factors on hospital adoption of technological and administrative innovation. Academic of Management of Journal, 24(4), 689-713.

Lawson, B., \& Samson D. (2001). Developing innovation capability in organizations: a dynamic capabilities approach. International Journal of Innovation Management, 5(3), 377-400.

Lee, H.S., \& Hong, S.A. (2014). Factors affecting hospital employees knowledge sharing intention and behavior, and innovation behavior. Osong Public Health Research Perspective, 5(3), 148-155.

López, O.H., Villegas, G.C., \& Rodriguez, J.R. (2017). Capacidades de innovación en el contexto de las organizaciones de salud y desarrollo de un modelo teórico. Gac Méd Caracas, 125(4),1-12.

Marsh, H.W., Hau, K.T., Balla, J.R., \& Grayson, D. (1998). Is more ever too much? The number of indicators per factor in confirmatory factor analysis. Multivariate Behavioral Research, 33, 181-220.

Martinez-Roman, J.A., Gamero J., \& Tamayo, J.A. (2011). Analysis of innovation in SMEs using an innovative capability-based non-linear model: A study in the province of Seville (Spain). Technovation, 31,459-475.

Mylopulos, M., \& Scardamalia. (2008). Doctors' perspectives on their innovations in daily practice: implications for knowledge building in health care. Medical Education, 42(10), 7581.

Naranjo, J.C., Jiménez, D. \& Sanz-Valle, V.R. (2015). Studying the links between organizational culture, innovation, and performance in Spanish companies. Revista Latinoamericana de Psicología, 48, 30-41.

Nonaka, I., \& Takeuchi, H. (1995). The Knowledge Creating Company. How Japanese Companies Create the Dynamics of Innovation. New York: Oxford University Press.

Omachunu, V., \& Einspruch, N. (2010). Innovation in Healthcare Delivery Systems: A Conceptual Framework. The Innovation Journal: The Public Sector Innovation Journal, 15(1), 1-20.

Parnaby, J., \& Towill D.R. (2008). Enabling innovation in health-care delivery. Health Services Management Research, 21, 141-154. 
Pen, D. X., Schroeder, R. G. \& Shah, R. (2008). Linking routines to operations capabilities: A new perspective. Journal of Operations Management, 26, 730-748.

Plsek, P. (2003). Complexity and the adoption of innovation in health care. Accelerating Quality Improvement in Health Care Strategies to Speed the Diffusion of Evidence-Based Innovations. National Institute for Health Care Management Foundation National Committee for Quality Health Care, Washington, D.C.

Salaman, J.G., \& Storey, J. (2002). Managers' theories about the process of innovation', Journal of Management Studies, 39(2), 147-165.

Schreyogg, G., \& Kliesch-Eberl, M. (2007). How dynamic can organizational capabilities be? Towards a dual-process model of capability dynamization. Strategic Management Journal, 28(9), 913933.

Schumacker, R.E., \& Lomax, R.G. (2010). Structural Equation Modeling, 3ra Edition. NewYork: Routledge Taylor \& Francis Group.

Sharma, S., Conduit, J., \& Hill, S. (2014). Organisational capabilities for customer participation in health care service innovation. Australasian Marketing Journal, 22,179-188.

Thakur, R., Hsu, S., \& Fontenot, G. (2012). Innovation in healthcare: Issues and future trends. Journal of Business Research, 65, 562-569.

Ugurluoglu, O., Aldogan, E.U., \& Dilmac, E. (2013). The impact of manager's perceptions of learning organisations on innovation in health care: sample of Turkey. International Journal Health Planning Management, 28, 158-168.

Vasquez, J. (2013). Modelos de ecuaciones estructurales en Psicología. Tesis de Maestría.

Villegas, G.C., Montes, J.M., \& López, O.H, (2016). Predictores de la capacidad de innovación en las organizaciones. Revista Espacios, 37(9), 3-10.

Yan, E. (2014). Finding knowledge paths among scientific disciplines. Journal of the Association for Information Science and Technology, 65(11), 2331-2347.

Yesil, S., Koska, A., \& Buyukbese, T. (2013). Knowledge Sharing Process, Innovation Capability and Innovation Performance: An Empirical Study. Social and Behavioral Sciences, 75, 217-225. 